\title{
SEM-EDS coating thickness assessment: an insight into the accuracy of Monte Carlo simulations carried out for TiN coatings using three different freeware graphical user interface
}

\author{
Juan P.N. Cruz ${ }^{1}$, Carlos M. Garzon ${ }^{1}$ and Abel. A.C. Recco ${ }^{2}$
}

${ }^{1}$ Departamento de Física, Universidad Nacional de Colombia, Bogotá, Distrito Capital de Bogota, Colombia, ${ }^{2}$ Departamento de Física, Universidade do Estado de Santa Catarina, Santa Catarina, Brazil

Stylus profilometry, quartz crystal microbalance, and cross-sectional microscopy [1] are widely used at both industrial and academic lab facilities for assessing the thickness of tribological nitride coatings. Energy dispersive spectroscopy (EDS) in the scanning electron microscope (SEM) allows a straightforward simultaneous characterization of film's elemental composition and thickness. Among the characterization methods above mentioned, SEM-EDS coating thickness assessment is cost-effective when a nondestructive and local (lateral resolution of the magnitude order of $1.0 \square \mathrm{m}$ ) analysis is aimed at.

Freeware graphical user interfaces (GUIs) for Monte Carlo (MC) simulation of the emission of characteristic $\mathrm{X}$-ray photons in SEM-EDS experiments are available [2,3]. They have been used for assessing EDS coating thickness via MC analysis of experimental EDS spectra [4]. In this contribution, the accuracy of MC predictions about EDS coating thickness assessment for TiN films magnetron sputtered onto either stainless steel, glass, or silicon is reported. Accuracy of predictions carried out using three different GUIs are compared among them, namely, Casino, DTSA-II, and MC-Xray. MC-appraised coating thicknesses are compared with reference values independently obtained by cross-sectional microscopy, and the percent discrepancy (predicted vs reference thicknesses) as a function of the nominal coating thickness and the microscope accelerating voltage $\left(\mathrm{V}_{0}\right)$ is studied. TiN coatings with increasing thicknesses (obtained by sputtering a Ti target for 15, 30, 60 and 90 minutes) are studied. We present in this contribution a straightforward analysis methodology where MC simulations are performed to find out, in the predicted EDS spectrum, with the coating thickness as a fitting parameter, the ratio between the strongest peak coming from the substrate (either $\mathrm{Fe}_{\mathrm{k}}$ or $\mathrm{Si}_{\mathrm{k}}$ ) and the $\mathrm{Ti}_{\mathrm{k}}$ peak (from the coating) which exactly match the concomitant ratio (afterwards called Intensity-ratio, $\mathrm{I}_{\mathrm{R}}$ ) in the experimental EDS spectrum. Figure 1 shows an example of the $\mathrm{I}_{\mathrm{R}}$ for an experimental EDS spectrum, the MCestimated coating thickness, the cross-sectional micrograph of the actual coating and the concomitant reference coating thickness. Figure 2.a shows the percent discrepancy between predictions carried out with Casino, DTSA-II, and MC-Xray, and Figure 2.b shows the percent discrepancy between MC-predicted (the averaged values from the three GUIs studied are presented) and the reference thicknesses. Further detailed analysis of the percent discrepancy as a function of $\mathrm{V}_{0}$ for TiN coatings which were grown for 15 minutes was carried out (not shown for simplicity). Discrepancy between MC-predicted and reference thicknesses significantly varied among the three pieces of software in the comparison, although, in general, none of that three software's outperformed the others. Most of the predicted thicknesses (90\% of the data analyzed) did not deviate more than $15 \%$ from the SEM-reference values, where the higher discrepancies were observed both at the lower accelerating voltages and the lower coating thicknesses. Eventual incidence of secondary fluorescence occurring at both coating-to-substrate interfaces and coating surfaces was qualitatively analyzed. An in-depth insight into the role of such secondary fluorescence on the impaired accuracy of thicknesses predicted for thinner coatings is presented. The role of the substrate type on the accuracy of MC-predicted thicknesses is discussed. 
(a) SEM micrographs

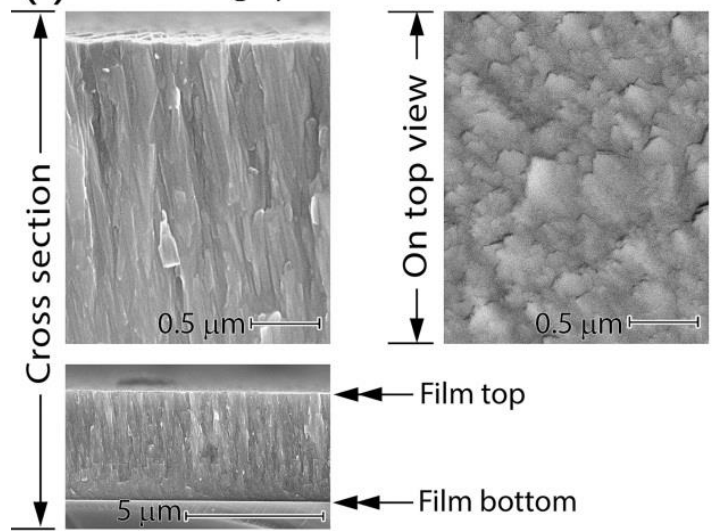

(b) EDS spectra

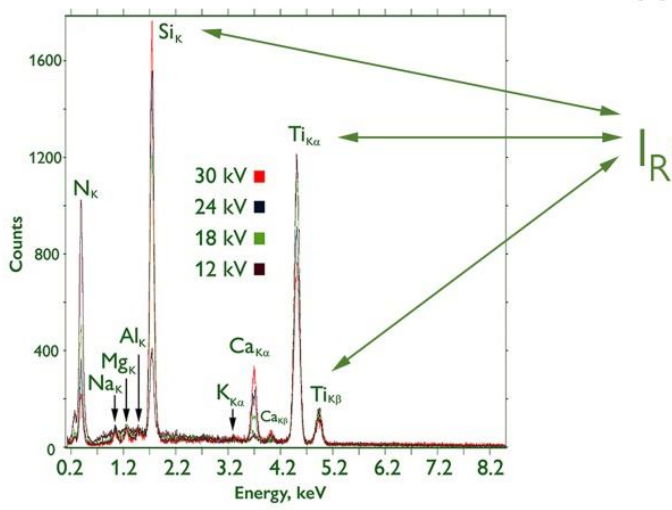

(c) Intensity-ratio, $I_{R}$

$R=\frac{\text { Intensity } \mathrm{Si}_{\mathrm{k}}}{\text { Intensity }\left(\mathrm{Ti}_{\mathrm{k \alpha}}+\mathrm{Ti}_{\mathrm{k} \beta}\right)}$

At fixed coating thickness, the higher $V_{0}$ the higher $I_{R}$.

Figure 1. Example of assessment of coating thickness from a SEM cross-sectional micrograph (a) and the concomitant assessment of IR for an experimental EDS spectrum(b) using the intensity-ratio defined in the inset (c). Secondary electrons. No chemical etching. TiN coating onto glass substrate. Sputtering time 90 minutes.
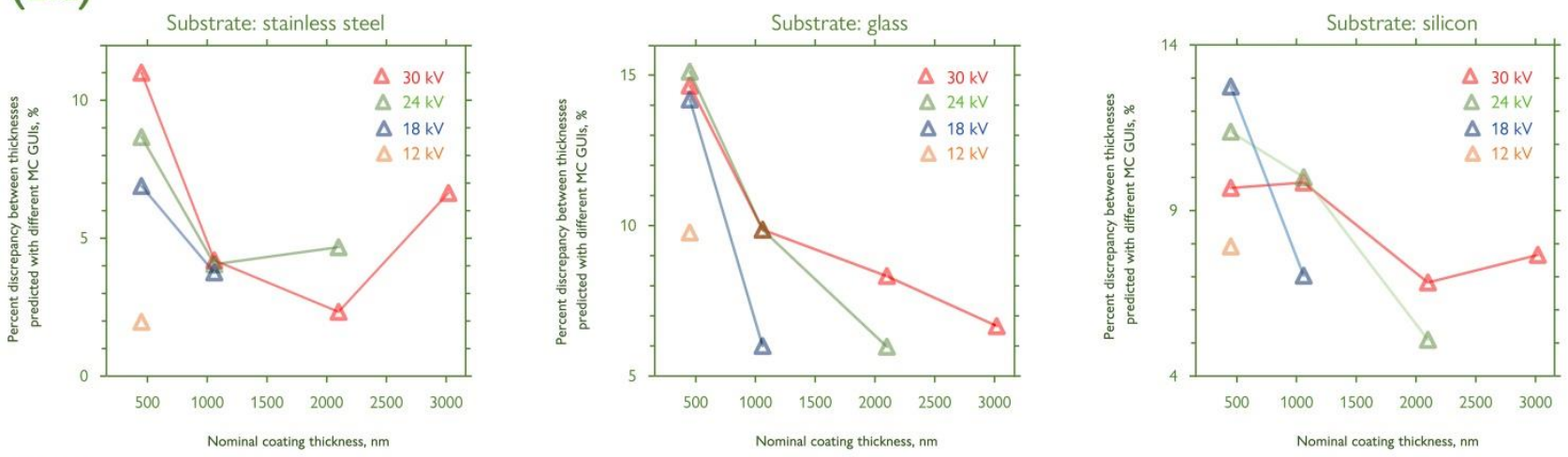

(2.b)
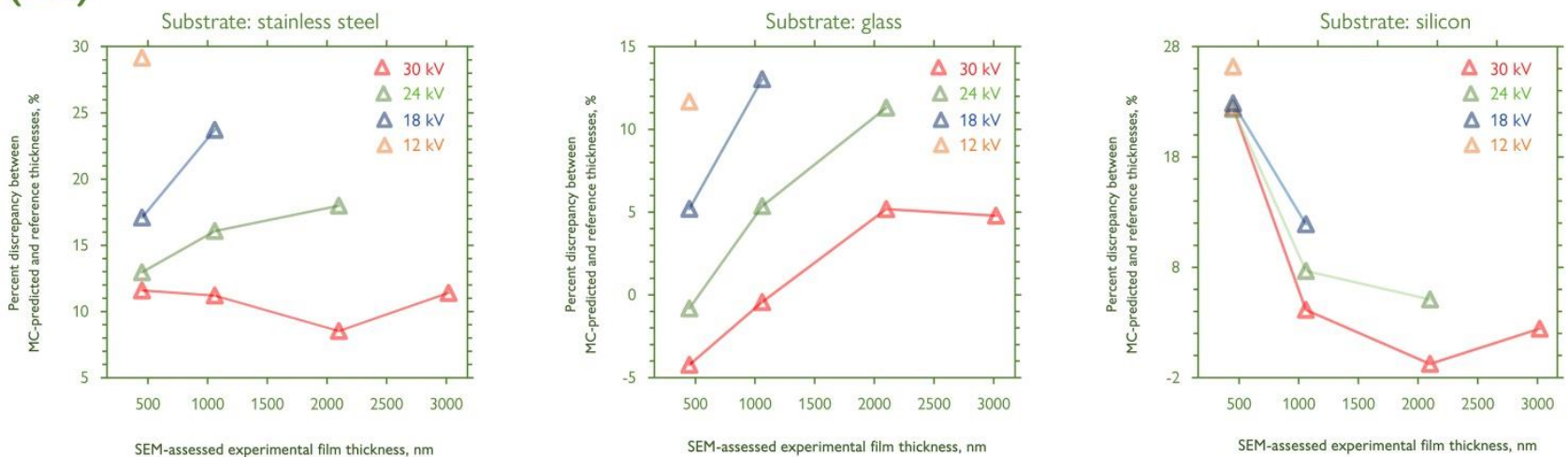

Figure 2. (a) Percent discrepancy between predictions carried out with Casino, DTSA-II, and MC-Xray. (b) Percent discrepancy between MC-predicted (the averaged values from the three GUIs studied are presented) and the reference thicknesses assessed by cross-sectional microscopy.

\section{References}

[1] Giurlani, W., Berretti, E., Innocenti, M. \& Lavacchi, A. (2020). Measuring the Thickness of Metal Films: A Selection Guide to the Most Suitable Technique. Mater. Proc. 2(1), 12. https://doi.org/10.3390/CIWC2020$\underline{06823}$ 
[2] Michaud, P. \& Gauvin, R. (2010), MC X-Ray, the Monte Carlo program for quantitative electron microscopy of real materials, Microsc Microanal, 16(S2), 278-279, https://doi.org/10.1017/S1431927610054668.

[3] Ritchie, N. W.M. (2009), Spectrum simulation in DTSA-II, Microsc Microanal, 15(5), 454-468, https://doi.org/10.1017/S1431927609990407.

[4] Matthews, M.B., Kearns, S.L. \& Buse, B. (2018), The accuracy of Al and Cu film thickness determinations and the implications for electron probe microanalysis. Microsc Microanal. 24(2), 83-92, https://doi.org/10.1017/S1431927618000193. 